\title{
SINGULAR RIGHT FOCAL BOUNDARY VALUE PROBLEM WITH GIVEN MAXIMAL VALUES
}

\author{
YU TIAN AND WEIGAO GE
}

\begin{abstract}
In this paper, we prove existence results for the singular problem $(-1)^{n-p}\left(\Phi_{m} x^{(n-1)}\right)^{\prime}$ $(t)=\mu f\left(t, x(t), \ldots, x^{(n-1)}(t)\right)$, for $0<t<1, x^{(i)}(0)=0, i=0,1, \ldots, p-1, x^{(i)}(1)=0, i=p$, $p+1, \ldots, n-1, \max \{x(t): t \in[0,1]\}=A$. The paper presents conditions which guarantee that for any $A>0$ there exists $\mu_{A}>0$ such that the above problem with $\mu=\mu_{A}$ has a solution $x \in C^{n-1}([0,1])$ with $\Phi_{m}\left(x^{(n-1)}\right) \in A C([0,1])$ which is positive on $(0,1)$. Here the positive Carathédory function $f$ may be singular at the zero value of all its phase variables. Proofs are based on the Leray-Schauder degree and Vitali's convergence theorem.
\end{abstract}

\section{Introduction}

The right focal boundary value problems has been widely studied by a number of authors in recent years. For details, see [1, 7, 8, 9, 10, 15] and the references therein. However the boundary value problems treated in the above mentioned references are not allowable to process singularity. For studies about higher-order singular boundary value problem, we refer to $[2,3,4,5,6,17]$.

Agarwal, O'Regan and Lakshmikantham studied the existence of solutions for right focal boundary value problem in [3]:

$$
\left\{\begin{array}{l}
(-1)^{n-p} y^{(n)}=\phi(t) f\left(t, y, \ldots, y^{(n-1)}\right), \quad n \geq 2, t \in(0,1) \\
y^{(i)}(0)=0, \quad 0 \leq i \leq p-1 \\
y^{(i)}(1)=0, \quad p \leq i \leq n-1
\end{array}\right.
$$

where $f \in C\left([0,1] \times(0, \infty)^{p},(0, \infty)\right), f\left(t, y_{0}, \ldots, y_{n-1}\right)$ may be singular at $y_{i}=0,0 \leq$ $i \leq p-1, \phi \in C(0,1)$ with $\phi>0$ on $(0,1)$ and $\phi \in L^{1}[0,1], \phi$ may be singular at $t=0$ and/or 1 . However, by assuming that $f$ has the following increasing condition

$$
\sum_{i=0}^{p-1} h_{i}\left(u_{i}\right) \leq f\left(t, u_{0}, \ldots, u_{p-1}\right) \leq \sum_{i=0}^{p-1} g_{i}\left(u_{i}\right)+r(u)
$$

Received April 25, 2005.

2000 Mathematics Subject Classification. 34B15, 34B16, 34B18.

Key words and phrases. Singular higher-order differential equation, regularization, Vitali's convergence theorem, right focal.

Supported by grant 10371006 from National Natural Sciences Foundation of P.R. China and grant 20050007011 from Foundation for PhD Specialities of Educational Department of P.R. China. 
on $[0,1] \times(0, \infty)^{p}$ with $h_{i}>0$ continuous and non-increasing on $(0, \infty)$ for each $i=$ $0, \ldots, p-1, g_{i}>0$ continuous and non-increasing on $(0, \infty)$ for each $i=0, \ldots, p-1$, and $r \geq 0$ continuous, nondecreasing on $[0, \infty)$, here $|u|=\max \left\{u_{0}, u_{1}, \ldots, u_{n-1}\right\}$.

$$
\int_{0}^{1} \phi(s) g_{i}\left(k_{i} s^{p-i}\right) d s<\infty \text { for each } i=0, \ldots, p-1
$$

here $k_{i}>0(i=0, \ldots, p-1)$ are constant, and

$$
\text { if } z>0 \text { satisfies } z \leq a_{0}+b_{0} r(z) \text { for constants } a_{0} \geq 0 \text { and } b_{0} \geq 0 \text {, }
$$

then there exists a constant $K$ (which may depend only on $a_{0}$ and $b_{0}$ ) with $z \leq K$.

The authors obtain an existence result. In fact, condition (1.4) implies the degree of variable $u$ in the term $r(u)$ must be smaller than 1 .

In [6], the singular problem $(-1)^{n} x^{(2 n)}(t)=\mu f\left(t, x, \ldots, x^{(2 n-2)}\right), x^{(2 j)}(0)=x^{(2 j)}(T)$ $=0,(0 \leq j \leq n-1), \max \{x(t): 0 \leq t \leq T\}=A$ depending on the parameter $\mu$ is considered. The existence of at least one positive solution was obtained under the assumption

$$
f\left(t, x_{0}, \ldots, x_{n-2}\right) \leq \phi(t)+\sum_{j=0}^{2 n-2} q_{j}(t) \omega_{j}\left(\left|x_{j}\right|\right)+\sum_{j=0}^{2 n-2} h_{j}(t)\left|x_{j}\right|^{\alpha_{j}}
$$

for a.e. $t \in J$ and for each $\left(x_{0}, \ldots, x_{2 n-2}\right) \in D$, where $\phi, h_{j} \in L_{1}(J)$ and $q_{j} \in L_{\infty}(J)$ are nonnegative, $\omega_{j}: R_{+} \rightarrow R_{+}$are non-increasing, $\alpha_{j} \in(0,1)$.

Motivated by the above results, we consider the right focal boundary value problem in the following form

$$
\begin{array}{r}
(-1)^{n-p}\left(\Phi_{m}\left(x^{(n-1)}\right)\right)^{\prime}(t)=\mu f\left(t, x(t), \ldots, x^{(n-1)}(t)\right), \quad 0<t<1, \\
x^{(i)}(0)=0, \quad i=0,1, \ldots, p-1, \quad x^{(i)}(1)=0, \quad i=p, p+1, \ldots, n-1 .
\end{array}
$$

Together with the boundary conditions (1.6), we discuss the condition

$$
\max \{x(t): t \in J\}=A,
$$

where $\Phi_{m} x:=|x|^{m-2} x, m>1, \Phi_{m^{\prime}}$ is the inverse operator of $\Phi_{m}$, where $\frac{1}{m}+\frac{1}{m^{\prime}}=$ $1, n \geq 2$.

Let $\bar{J}=[0,1], R_{-}=(-\infty, 0), R_{+}=(0, \infty), R_{0}=R \backslash\{0\}$,

$$
D= \begin{cases}\underbrace{R_{+} \times \cdots \times R_{+}}_{p} \times \underbrace{R_{+} \times R_{-} \times \cdots \times R_{+}}_{n-p}, & n-p=2 k+1, \\ \underbrace{R_{+} \times \cdots \times R_{+}}_{p} \times \underbrace{R_{+} \times R_{-} \times \cdots \times R_{-}}_{n-p}, & n-p=2 k .\end{cases}
$$

Nonlinearity term $f$ satisfies local Carathédory conditions on $J \times D(f \in \operatorname{Car}(J \times D))$ and may be singular at the zero value of all its phase variables. By using Leray-Schauder 
degree theory we get a new result on the existence of solutions to boundary value problem (1.5)-(1.7). The method of obtaining priori bound of solution is different from $[3$, 6] In addition, the maximum degree of some variables among $x_{0}, \ldots, x_{n-1}$ in function $f\left(t, x_{0}, \ldots, x_{n-1}\right)$ are allowable to be 1 .

Let $A \in R^{+}$. By a solution of BVP (1.5)-(1.7) we understand a function $x \in A C^{n-1}(J)$ (i.e., $x$ has an absolutely continuous $(n-1)$ st derivative on $J$ ) such that

(i) $x^{(i)}(t)>0$ on $(0,1]$ for $i=0, \ldots, p-1$ and $(-1)^{2 n-p-i} x^{(i)}(t)>0$ on $[0,1)$ for $i=p, \ldots, n-1$,

(ii) $x$ satisfies boundary conditions (1.6)(1.7),

(iii) there exists $\mu_{A} \in R_{+}$such that $x$ fulfills (1.5) with $\mu=\mu_{A}$ for a.e. $t \in J$.

By a solution of BVP (1.5), (1.6) we understand a function $x \in A C^{2 n-1}(J)$ such that $x^{(i)}(t)>0$ on $(0,1]$ for $i=0, \ldots, p-1$ and $(-1)^{n-i+1} x^{(2 n-p-i)}(t)>0$ on $[0,1)$ for $i=p, \ldots, n-1, x$ satisfies boundary conditions (1.6) and (1.5) holds a.e. $t \in J$.

The purpose of this paper is to give conditions which guarantee the existence of a solution to BVP (1.5)-(1.7) for each given $A \in R_{+}$.

From now on, $\|x\|=\max \{|x(t)|: t \in J\},\|x\|_{1}=\int_{0}^{1}|x(t)| d t$ and $\|x\|_{\infty}=e s s \max \{$ $|x(t)|: 0 \leq t \leq 1\}$ stands for the norm in $C^{0}(J), L_{1}(J)$, and $L_{\infty}(J)$, respectively. For any measurable set $\mathcal{M} \subset R, \mu(\mathcal{M})$ denotes the Lebesgue measure of $\mathcal{M}$.

The assumptions imposed upon the function $f$ in (1.5) are listed as follows:

$\left(H_{1}\right) f \in \operatorname{Car}(J \times D)$ and there exists nonnegative functions $\phi \in L_{1}(J), q_{i} \in L_{\infty}(J)$, and continuous functions $g_{i}:[0,1] \times R^{n} \rightarrow R^{+}(i=0, \ldots, n-1)$ and non-increasing nonnegative continuous function $\omega_{i}: R_{+} \rightarrow R_{+}$such that for $(t, x) \in J \times D$,

$$
f\left(t, x_{0}, \ldots, x_{n-1}\right)=\phi(t)+\sum_{i=0}^{n-1} q_{i}(t) \omega_{i}\left(\left|x_{i}\right|\right)+\sum_{i=0}^{n-1} g_{i}\left(t, x_{i}\right),
$$

where

$$
\lim _{\left|x_{i}\right| \rightarrow \infty} \sup _{t \in[0,1]} \frac{g_{i}\left(t, x_{i}\right)}{\left(\Phi_{m}\left(\left|x_{i}\right|\right)\right)^{k_{i}}}=\alpha_{i} \geq 0, \quad k_{i} \text { are any constants in }(0,1), i=0, \ldots, p-1,
$$

and

$$
\lim _{\left|x_{i}\right| \rightarrow \infty} \sup _{t \in[0,1]} \frac{g_{i}\left(t, x_{i}\right)}{\Phi_{m}\left(\left|x_{i}\right|\right)}=\beta_{i} \geq 0, \quad i=p, \ldots, n-1,
$$

and $\omega_{i}$ satisfies

$$
\int_{0}^{1} \omega_{i}\left(s^{p-i}\right) d s<\infty, 0 \leq i \leq p-1, \quad \int_{0}^{1} \omega_{i}\left(P_{i}(s)\right) d s<\infty, p \leq i \leq n-1,
$$

where

$$
P_{i}(t)=\frac{1}{(n-2-i) !} \int_{t}^{1}(\theta-t)^{n-2-i} \Phi_{m^{\prime}}\left(\int_{\theta}^{1} \phi(r) d r\right) d \theta
$$

and there exists $\lambda>0$ such that

$$
\omega_{i}(x y) \leq \lambda \omega_{i}(x) \omega_{i}(y) \text { for } x, y \in(0, \infty) .
$$


The paper is organized as follows. Section 2 presents the priori bound of BVP (1.5)(1.7). Besides, we prove that some sets of functions containing solutions of our auxiliary regular BVPs are uniformly absolutely continuous on $J$. Section 3 deals with auxiliary regular BVPs of problem (1.5), (1.6), (1.7). First we prove the existence of solution by applying the Borsuk antipodal theorem and the Leray-Schauder degree (see, e.g. [12]). Then we prove the existence of solution for problem (1.5), (1.6), (1.7). Proof is based on the Arzelà-Ascoli theorem and the Vitali's convergence theorem, see, e.g. [11, 12, 14].

\section{Auxiliary Results}

Lemma 2.1. If $y$ is a solution of $B V P(1.5),(1.6)$, then $y(t)$ is a fixed point of the operator

$$
(T y)(t)=(-1)^{n-p-1} \int_{0}^{1} G(t, s) \Phi_{m^{\prime}}\left(\int_{s}^{1} f\left(\theta, y(\theta), \ldots, y^{(n-1)}(\theta)\right) d \theta\right) d s
$$

where $G(t, s)$ is the Green's function of the following BVP

$$
\left\{\begin{array}{l}
x^{(n-1)}(t)=0, \quad t \in(0,1), \\
x^{(i)}(0)=0, \quad i=0, \ldots, p-1, \quad x^{(i)}(1)=0, \quad i=p, \ldots, n-2,
\end{array}\right.
$$

and $G(t, s)$ can be expressed as

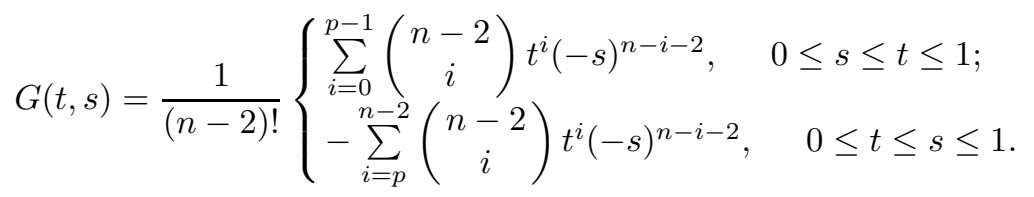

Furthermore,

$$
\begin{aligned}
& (-1)^{n-p-1} \frac{\partial^{i}}{\partial t^{i}} G(t, s) \geq 0, i=0, \ldots, p-1 \\
& (-1)^{n-i-1} \frac{\partial^{i}}{\partial t^{i}} G(t, s) \geq 0, \quad i=p, \ldots, n-2,(t, s) \in J \times J .
\end{aligned}
$$

Proof. By integrating the equation in (1.5) from $t \in[0,1)$ to 1 and using $x^{(n-1)}(1)=$ 0 , we obtain that

$$
(-1)^{n-p} \Phi_{m}\left(y^{(n-1)}(t)\right)=-\int_{t}^{1} f\left(\theta, y(\theta), \ldots, y^{(n-1)}(\theta)\right) d \theta
$$

i.e.

$$
y^{(n-1)}(t)=(-1)^{n-p-1} \Phi_{m^{\prime}}\left(\int_{t}^{1} f\left(\theta, y(\theta), \ldots, y^{(n-1)}(\theta)\right) d \theta\right)
$$


By [15] we have the result is true.

Remark 2.1. It follows from (2.1) (2.2) that

$$
\left\{\begin{array}{l}
y^{(i)}(t)>0, \quad i=0, \ldots, p-1, \quad t \in(0,1] \\
(-1)^{i-p} y^{(i)}(t)>0, \quad i=p, \ldots, n-1, \quad t \in[0,1) .
\end{array}\right.
$$

As in [5], for each $m \in N$, define $\mathcal{X}_{m}, \varphi_{m} \in C^{0}(R), R_{m} \subset R$ and $f_{m} \in \operatorname{Car}\left(J \times R^{n}\right)$ by the formulas

$$
\begin{gathered}
\mathcal{X}_{m}(u)=\left\{\begin{array}{ll}
u, & \text { for } u \geq \frac{1}{m}, \\
\frac{1}{m}, & \text { for } u<\frac{1}{m},
\end{array} \quad \varphi_{m}(u)= \begin{cases}-\frac{1}{m}, & \text { for } u>-\frac{1}{m}, \\
u, & \text { for } u \leq-\frac{1}{m},\end{cases} \right. \\
\tau_{m}(u)= \begin{cases}\mathcal{X}_{m}(u), & \text { for } n-p=2 k+1, \\
\varphi_{m}(u), & \text { for } n-p=2 k,\end{cases}
\end{gathered}
$$

and

$$
\begin{aligned}
& f_{m}\left(t, x_{0}, \ldots, x_{n-1}\right) \\
= & \phi(t)+\sum_{i=0}^{p-1} q_{i}(t) \omega_{i}\left(\left|\mathcal{X}_{m}\left(x_{i}\right)\right|\right)+q_{p}(t) \omega_{p}\left(\left|\mathcal{X}_{m}\left(x_{p}\right)\right|\right)+q_{p+1}(t) \omega_{p+1}\left(\left|\varphi_{m}\left(x_{p+1}\right)\right|\right) \\
& +\cdots+q_{n-1}(t) \omega_{n-1}\left(\left|\tau_{m}\left(x_{n-1}\right)\right|\right)+\sum_{i=0}^{n-1} g_{i}\left(t, x_{i}\right)
\end{aligned}
$$

for $\left(t, x_{0}, \ldots, x_{n-1}\right) \in J \times R^{n}$. Hence

$$
\begin{aligned}
0<\phi(t) & \leq f_{m}\left(t, x_{0}, \ldots, x_{n-1}\right) \\
& \leq \phi(t)+\sum_{i=0}^{n-1} q_{i}(t) \omega_{i}\left(\left|x_{i}\right|\right)+\sum_{i=0}^{n-1} g_{i}\left(t, x_{i}\right)
\end{aligned}
$$

for a.e. $t \in J$ and each $\left(x_{0}, \ldots, x_{n-1}\right) \in R_{0}^{n}$.

Consider auxiliary regular differential equation

$$
\left(\Phi_{m} x^{(n-1)}\right)^{\prime}(t)=\mu f_{m}\left(t, x(t), \ldots, x^{(n-1)}(t)\right)
$$

depending on the parameters $\mu \in R$ and $m \in N$.

Lemma 2.2. Let $m \in N$, then

$$
\begin{aligned}
& x^{(i)}(t) \geq t^{p-i} \Gamma, \quad i=0, \ldots, p-1 ; \quad(-1)^{2 n-p-i} x^{(i)}(t) \geq P_{i}(t), \quad i=p, \ldots, n-1, \quad \text { (2.6) } \\
& \text { on } J \text { for any solution } x \text { of } B V P(2.6), \quad(1.6), \text { where } \Gamma=(-1)^{n-p-1} \int_{0}^{1} G(1, s) \Phi_{m^{\prime}} \\
& \left(\int_{s}^{1} \phi(\theta) d \theta\right) d s \text {. }
\end{aligned}
$$


Proof. By [2] we have

$$
x^{(i)}(t) \geq t^{p-i} x^{(i)}(1) \text { for } t \in J, i=0, \ldots, p-1 .
$$

Applying the inequality $\left\|x^{(i)}\right\| \geq\|x\|, i=0, \ldots, n-1$, and (2.1), (2.2) to (2.7) we get

$$
\begin{aligned}
x^{(i)}(t) \geq t^{p-i}\|x\| & =t^{p-i} x(1) \geq(-1)^{n-p-1} t^{p-i} \int_{0}^{1} G(1, s) \Phi_{m^{\prime}}\left(\int_{s}^{1} \mu \phi(\theta) d \theta\right) d s \\
& =\Phi_{m^{\prime}}(\mu) t^{p-i} \Gamma
\end{aligned}
$$

for $t \in J, i=0, \ldots, p-1$.

On the other hand, by $(2.3)$ we have $(-1)^{n-p} x^{(n)}(t) \geq \phi(t), t \in J$. Integrating the above inequality from $t$ to 1 , we get step by step

$$
(-1)^{2 n-p-i} x^{(i)}(t) \geq \int_{t}^{1} \frac{(\theta-t)^{n-i-2}}{(n-i-2) !} \Phi_{m^{\prime}}\left(\int_{\theta}^{1} \phi(r) d r\right) d \theta=P_{i}(t), \quad i=p, \ldots, n-1 .
$$

Lemma 2.3. Suppose that assumption $\left(H_{1}\right)$ is satisfied, $m \in N$ and $A \in R^{+}$. Denote $\mu_{*}=\Phi_{m}\left(\frac{A}{\Gamma}\right)$. Then there is no solution in $B V P(2.5),(1.6),(1.7)$ for $\mu>\mu_{*}$.

Proof. Suppose $x(t)$ is a solution of BVP (2.5), (1.6), (1.7). By (2.1) and (2.4) we have

$$
\begin{aligned}
x(t) & =(-1)^{n-p-1} \int_{0}^{1} G(t, s) \Phi_{m^{\prime}}\left(\mu \int_{s}^{1} f_{m}\left(r, x(r), \ldots, x_{n-1}(r)\right) d r\right) d s \\
& \geq(-1)^{n-p-1} \int_{0}^{1} G(t, s) \Phi_{m^{\prime}}\left(\mu \int_{s}^{1} \phi(r) d r\right) d s,
\end{aligned}
$$

i.e.

$$
\begin{aligned}
\max \{x(t): t \in J\} & \geq(-1)^{n-p-1} \Phi_{m^{\prime}}(\mu) \max _{t \in J} \int_{0}^{1} G(t, s) \Phi_{m^{\prime}}\left(\int_{s}^{1} \phi(r) d r\right) d s \\
& >(-1)^{n-p-1} \Phi_{m^{\prime}}\left(\mu_{*}\right) \int_{0}^{1} G(1, s) \Phi_{m^{\prime}}\left(\int_{s}^{1} \phi(r) d r\right) d s \\
& =\Phi_{m^{\prime}}\left(\mu_{*}\right) \Gamma=A
\end{aligned}
$$

which contradicts to (1.7).

Lemma 2.4. Suppose $0<u \in L^{1}[0, T], 0 \leq \psi \in L^{\infty}[0, T]$ and

$$
u(t) \leq K+\int_{t}^{T} u(s) \psi(s) d s, t \in[0, T], K>0
$$

Then $u(t) \leq K \exp \int_{t}^{T} \psi(s) d s, \quad \forall t \in[0, T]$. 
Proof. Let $G(t)=K+\int_{t}^{T} u(s) \psi(s) d s$, then $G^{\prime}(t)=-u(t) \psi(t) \geq-\psi(t) G(t)$, i.e.

$$
\frac{G^{\prime}(t)}{G(t)} \geq-\psi(t)
$$

Integrating the above inequality from $t$ to $T$ we have

$$
\ln K-\ln G(t) \geq-\int_{t}^{T} \psi(s) d s
$$

i.e. $G(t) \leq K \exp \int_{t}^{T} \psi(s) d s$. Then $u(t) \leq G(t) \leq K \exp \int_{t}^{T} \psi(s) d s$.

Lemma 2.5. Let assumption $\left(H_{1}\right)$ be satisfied and $A \in R_{+}$. Then there exists a positive constant $P$ depending only on $A$ such that for any solution $x$ of $B V P(2.5),(1.6)$ with a $\mu \in R_{+}$satisfying

$$
\max \{x(t): t \in J\}=\lambda A, \quad \lambda \in(0,1]
$$

we have

$$
\mu \leq \mu_{*} \text { and }\left\|x^{(j)}\right\| \leq P \text { for } 0 \leq j \leq n-1,
$$

where $\mu_{*}$ is defined in Lemma 2.3 .

Proof. Let $x$ be a solution of BVP (2.5), (1.6) with $\mu \in R_{+}$satisfying (2.8) for some $\lambda \in(0,1]$. Then by Lemma $2.3, \mu \leq \Phi_{m}(\lambda) \mu_{*}$ and so $\mu \leq \mu_{*}$.

Following we will show $\left\|x^{(j)}\right\| \leq P, j=0, \ldots, n-1$. We finish the proof by three steps.

Step 1. It follows from boundary condition that

$$
\begin{gathered}
x^{(i)}(t)=\int_{0}^{t} \frac{(t-s)^{p-i-1}}{(p-i-1) !}\left(\int_{s}^{1} \frac{(\theta-s)^{n-p-2}}{(n-p-2) !}\left|x^{(n-1)}(\theta)\right| d \theta\right) d s, \quad i=0, \ldots, p-1 . \\
(-1)^{2 n-p-i} x^{(i)}(t)=\int_{t}^{1} \frac{(\theta-t)^{n-i-2}}{(n-i-2) !}\left|x^{(n-1)}(\theta)\right| d \theta, \quad i=p, \ldots, n-2 .
\end{gathered}
$$

It follows from (2.11) that

$$
\left|x^{(i)}(t)\right| \leq \frac{(1-t)^{n-i-1}}{(n-i-1) !}\left|x^{(n-1)}(t)\right|, \quad i=p, \ldots, n-1 .
$$

From (2.10) we have

$$
\left\|x^{(i)}\right\| \leq \frac{1}{(p-i-1) !(n-p-1) !}\left\|x^{(n-1)}\right\|, \quad i=0, \ldots, p-1 .
$$

Step 2. Prove $\left|x^{(n-1)}(t)\right| \leq P, t \in[0,1]$. 
For any small $\varepsilon>0$, there is $\delta>0$ so that

$\left|g_{i}\left(t, x_{i}\right)\right|<\left(\alpha_{i}+\varepsilon\right)\left(\Phi_{m}\left(\left|x_{i}\right|\right)\right)^{k_{i}}$ uniformly for $t \in[0,1], k_{i} \in(0,1)$ and $\left|x_{i}\right|>\delta$, $i=0, \ldots, p-1$,

and

$\left|g_{i}\left(t, x_{i}\right)\right|<\left(\beta_{i}+\varepsilon\right) \Phi_{m}\left(\left|x_{i}\right|\right)$ uniformly for $t \in[0,1]$, and $\left|x_{i}\right|>\delta, i=p, \ldots, n-1$.

Let, for $i=0, \ldots, n-1$,

$$
\begin{gathered}
\Delta_{1, i}=\left\{t: t \in[0,1],\left|x_{i}(t)\right| \leq \delta\right\}, \\
\Delta_{2, i}=\left\{t: t \in[0,1],\left|x_{i}(t)\right|>\delta\right\}, \\
g_{\delta, i}=\max _{t \in[0,1],\left|x_{i}\right| \leq \delta} g_{i}\left(t, x_{i}\right) .
\end{gathered}
$$

For some $m>0, t \in[0,1]$,

$$
(-1)^{n-p}\left(\Phi_{m} x^{(n-1)}\right)^{\prime}(t)=\mu f_{m}\left(t, x(t), \ldots, x_{n-1}(t)\right) .
$$

Integrating the above equality from $t$ to 1 , noticing Lemma 2.2, (1.9), (1.10), (2.4) and (2.12) (2.13) we have

$$
\begin{aligned}
\Phi_{m}\left(\left|x^{(n-1)}(t)\right|\right) \leq & \mu_{*} \int_{t}^{1}\left[\phi(s)+\sum_{i=0}^{p-1} q_{i}(s) \omega_{i}\left(s^{p-i} \Gamma\right)+\sum_{i=p}^{n-1} q_{i}(s) \omega_{i}\left(P_{i}(s)\right)\right] d s \\
& +\sum_{i=0}^{n-1} \int_{\delta_{1, i} \cap[t, 1]} g_{i}\left(s, x^{(i)}(s)\right) d s+\sum_{i=0}^{n-1} \int_{\delta_{2, i} \cap[t, 1]} g_{i}\left(s, x^{(i)}(s)\right) d s \\
\leq & \mu_{*}\left(\Lambda+\sum_{i=0}^{n-1} g_{\delta, i}+\sum_{i=0}^{n-1} \int_{\delta_{2, i} \cap[t, 1]} g_{i}\left(s, x^{(i)}(s)\right) d s\right) \\
\leq & \mu_{*}\left[\Lambda+\sum_{i=0}^{n-1} g_{\delta, i}+\sum_{i=0}^{p-1}\left(\alpha_{i}+\varepsilon\right)\left(\Phi_{m}\left(\frac{\left|x^{(n-1)}(0)\right|}{(p-i-1) !(n-p-1) !}\right)\right)^{k_{i}}\right. \\
& \left.+\int_{t}^{1} \sum_{i=p}^{n-1}\left(\beta_{i}+\varepsilon\right) \Phi_{m}\left(\frac{(1-s)^{n-i-1}}{(n-i-1) !}\right) \Phi_{m}\left(\left|x^{(n-1)}(s)\right|\right) d s\right],
\end{aligned}
$$

i.e.

$$
\left|\Phi_{m}\left(x^{(n-1)}(t)\right)\right| \leq\left(C+D\left(\Phi_{m}\left(\left|x^{(n-1)}(0)\right|\right)\right)^{k_{i}}\right)+\int_{t}^{1} E(s) \Phi_{m}\left(\left|x^{(n-1)}(s)\right|\right) d s .
$$

where

$$
\Lambda=\int_{0}^{1}\left[\phi(s)+\sum_{i=0}^{p-1}\left\|q_{i}\right\|_{\infty} \lambda \omega_{i}\left(s^{p-i}\right) \omega_{i}(\Gamma)+\sum_{i=p}^{n-1}\left\|q_{i}\right\|_{\infty} \omega_{i}\left(P_{i}(s)\right)\right] d s
$$




$$
\begin{gathered}
C=2 \mu_{*}\left[\Lambda+\sum_{i=0}^{n-1} g_{\delta, i}\right] \\
D=2 \mu_{*} \sum_{i=0}^{p-1}\left(\alpha_{i}+\varepsilon\right)\left(\Phi_{m}\left(\frac{1}{(p-i-1) !(n-p-1) !}\right)\right)^{k_{i}}, \\
E(t)=2 \mu_{*} \sum_{i=p}^{n-1}\left(\beta_{i}+\varepsilon\right) \Phi_{m}\left(\frac{(1-t)^{n-i-1}}{(n-i-1) !}\right) .
\end{gathered}
$$

By Lemma 2.4 and keep in mind $k_{i} \in(0,1)$, so there exists $P$ (which does not independent on $\lambda$ ) such that $\left|x^{(n-1)}(0)\right|=\left\|x^{(n-1)}\right\| \leq P$.

Step 3. Prove $\left\|x^{(i)}\right\| \leq P$ for $i=0,1, \ldots, n-1$.

By (2.12) (2.15) and Step 2, we have

$$
\begin{aligned}
& \left\|x^{(i)}\right\| \leq \frac{P}{(n-i-1) !} \leq P, \quad \text { for } i=p, \ldots, n-2, \\
& \left\|x^{(i)}\right\| \leq \frac{P}{(n-p-1) !(p-i-1) !} \leq P, \text { for } i=0, \ldots, p-1 .
\end{aligned}
$$

Thus $\left\|x^{(i)}\right\| \leq P$ for $i=0,1, \ldots, n-1$.

Lemma 2.6. Let assumption $\left(H_{1}\right)$ be satisfied and $A \in R_{+}$. Let $B V P(2.5),(1.6)$, (1.7) has a solution $x_{m}$ for each $m \in N$ with $\mu=\mu_{m}$ in (2.5). Then the sequence

$$
\left\{\mu_{m} f_{m}\left(t, x_{m}(t), \ldots, x_{m}^{(n-1)}(t)\right)\right\} \subset L_{1}(J)
$$

is uniformly absolutely continuous on $J$, that is for each $\varepsilon>0$ there exists $\delta>0$ such that

$$
\mu_{m} \int_{\mathcal{M}} f_{m}\left(t, x_{m}(t), \ldots, x_{m}^{(n-1)}(t)\right) d t<\varepsilon
$$

for any measurable set $\mathcal{M} \subset J, \mu(\mathcal{M})<\delta$.

Proof. With respect to (2.5) and properties of measurable sets, it is sufficient to verify that for every $\varepsilon>0$, there exists $\delta>0$ such that for any at most countable set $\left\{\left(a_{j}, b_{j}\right)\right\}_{j \in J}$ of mutually disjoint intervals $\left\{\left(a_{j}, b_{j}\right)\right\}_{j \in J}$ with $\sum_{j \in J}\left(b_{j}-a_{j}\right)<\delta$, we have for each $m \in N$,

$$
\sum_{j \in J} \int_{a_{j}}^{b_{j}}\left[\phi(t)+\sum_{i=0}^{n-1} q_{i}(t) \omega_{i}\left(\left|x_{m}^{(i)}\right|\right)+\sum_{i=0}^{n-1} g_{i}\left(t, x^{(i)}(t)\right)\right] d t<\varepsilon .
$$

By Lemma 2.2 we have

$$
\begin{aligned}
x_{m}^{(i)}(t) & \geq t^{p-i} \Phi(t), \quad i=0, \ldots, p-1, \quad t \in J \\
\left|x_{m}^{(i)}(t)\right| & \geq P_{i}(t), \quad i=p, \ldots, n-1, \quad t \in J
\end{aligned}
$$


In addition by Lemma 2.4

$$
\left\|x_{m}^{(i)}\right\| \leq P, \quad i=0, \ldots, p-1 .
$$

From (1.12), (2.16), (2.17) we have

$$
\begin{aligned}
& \sum_{j \in J} \int_{a_{j}}^{b_{j}}\left[\phi(t)+\sum_{i=0}^{n-1} q_{i}(t) \omega_{i}\left(\left|x_{m}^{(i)}\right|\right)+\sum_{i=0}^{n-1} g_{i}\left(t, x^{(i)}(t)\right)\right] d t \\
\leq & \sum_{j \in J} \int_{a_{j}}^{b_{j}}\left[\phi(t)+\sum_{i=0}^{p-1} q_{i}(t) \lambda \omega_{i}\left(t^{p-i}\right) \omega_{i}(\Gamma)+\sum_{i=p}^{n-1} q_{i}(t) \omega_{i}\left(P_{i}(t)\right)+\sup _{(t, x) \in[0,1] \times[0, P]} g_{i}\left(t, x_{i}\right)\right] d t .
\end{aligned}
$$

By $\left(H_{1}\right)$, we know that $\phi, h_{i} \in L_{1}(J), q_{i} \in L_{\infty}(J), \int_{0}^{1} \omega_{i}\left(t^{p-i}\right) d t<\infty, i=0, \ldots, p-$ $1, \int_{0}^{1} \omega_{j}\left(P_{j}(s)\right) d s<\infty, j=p, \ldots, n-1$. Consequently, for each $\varepsilon>0$ there exists $\delta>0$ such that for any at most countable set $\left\{\left(a_{j}, b_{j}\right)\right\}_{j \in J}$ of mutually disjoint intervals $\left(a_{j}, b_{j}\right) \subset J$ with $\sum_{j \in J}\left(b_{j}-a_{j}\right)<\delta$. So $(2.17)$ holds.

\section{Existence results}

Theorem 3.1. Suppose that the assumption $\left(H_{1}\right)$ is satisfied and $A \in R_{+}$. Then for each $m \in N$ there exists a solution $x_{m}$ of $B V P(2.5),(1.6),(1.7)$ with $\mu=\mu_{m}$ in (2.5), and

$$
\left\|x_{m}^{(j)}\right\| \leq P \text { for } m \in N, j=0, \ldots, n-1
$$

and

$$
0<\mu_{m} \leq \mu_{*}
$$

Proof. Fix $m \in N$. Set

$$
\Omega=\left\{(x, \mu):(x, \mu) \in C^{n}(J) \times R,\left\|x^{(j)}\right\|<P+1 \text { for } j=0, \ldots, n-1,|\mu|<\mu_{*}+1\right\} .
$$

Then $\Omega$ is a bounded, open and symmetric with respect to $(0,0)$ subset of the Banach space $C^{n}(J) \times R$ endowed with the norm $\|(x, \mu)\|=\sum_{i=0}^{n-1}\left\|x^{(j)}\right\|+|\mu|$. Define the operator $\mathcal{F}_{1}: \bar{\Omega} \rightarrow C^{n}(R) \times R$ by

$$
\begin{aligned}
\mathcal{F}_{1}(x, \mu)= & \left((-1)^{n-p-1} \int_{0}^{1} G(t, s) \Phi_{m^{\prime}}\left(\mu \int_{s}^{1} f_{m}\left(r, x(r), \ldots, x^{(n-1)}(r)\right) d r\right) d s,\right. \\
& \max \{x(t): t \in J\}+\min \{x(t): t \in J\}+\mu),
\end{aligned}
$$

where $G$ is defined in Lemma 2.1. We first show that

$$
D\left(\mathcal{I}-\mathcal{F}_{1}, \Omega, 0\right) \neq 0
$$


where $D$ stands for the Leray-Schauder degree and $\mathcal{I}$ is the identity operator on $C^{n}(J) \times$ $R$. To prove (3.3) we define the operator $\mathcal{H}:[0,1] \times \bar{\Omega} \rightarrow C^{n-1}(J) \times R$,

$$
\begin{aligned}
\mathcal{H}(\lambda, x, \mu)= & \left((-1)^{n-p-1} \int_{0}^{1} G(t, s) \Phi_{m^{\prime}}\left[\mu(1-\lambda)+\mu \lambda \int_{s}^{1} f_{m}\left(r, x(r), \ldots, x^{(n-1)}(r)\right) d r\right] d s,\right. \\
& \lambda[\max \{x(t: t \in J)\}+\min \{x(t): t \in J\}]+(1-\lambda) x(1 / 2)+\mu) .
\end{aligned}
$$

Then

$$
\mathcal{H}(0,-x,-\mu)=\left(-(-1)^{n-p-1} \int_{0}^{1} G(t, s) \Phi_{m^{\prime}}(\mu) d s,-x(1 / 2)-\mu\right)=-\mathcal{H}(0, x, \mu)
$$

for $(x, \mu) \in \bar{\Omega}$ and so $\mathcal{H}$ is an odd operator. Due to the fact that $f_{m} \in \operatorname{Car}\left(J \times R^{n-1}\right)$, $\mathcal{H}$ is a compact operator. Assume that $\mathcal{H}\left(\lambda_{0}, x_{0}, \mu_{0}\right)=\left(x_{0}, \mu_{0}\right)$ for some $\lambda_{0} \in[0,1]$ and $\left(x_{0}, \mu_{0}\right) \in \partial \Omega$. Then

$$
x_{0}(t)=(-1)^{n-p-1} \int_{0}^{1} G(t, s) \Phi_{m^{\prime}}\left[\mu\left(1-\lambda_{0}\right)+\mu \lambda_{0} \int_{s}^{1} f_{m}\left(r, x_{0}(r), \ldots, x_{0}^{(n-1)}(r)\right) d r\right] d s
$$

for $t \in J$ and

$$
\lambda_{0}\left[\max \left\{x_{0}(t): t \in J\right\}+\min \left\{x_{0}(t): t \in J\right\}\right]+\left(1-\lambda_{0}\right) x_{0}(1 / 2)=0 .
$$

Also from (2.3) it follows that

$$
x_{0}(0)=0, x_{0}^{\prime}(t) \geq 0, t \in J
$$

So $x_{0}(t)>0$ for $t \in(0,1)$ and $\min \left\{x_{0}(t): t \in J\right\}=0$. Therefore

$$
\begin{aligned}
& \lambda_{0}\left[\max \left\{x_{0}(t): t \in J\right\}+\min \left\{x_{0}(t): t \in J\right\}\right]+\left(1-\lambda_{0}\right) x_{0}(1 / 2) \\
= & \lambda_{0} \max \left\{x_{0}(t): t \in J\right\}+\left(1-\lambda_{0}\right) x_{0}(1 / 2)>0,
\end{aligned}
$$

contrary to (3.5). If $\mu_{0}<0$, then $x_{0}(t)<0$ for $t \in(0,1)$. By $(2.3) x_{0}(0)=0, x_{0}^{\prime}(0) \leq$ $0, t \in J$, so $\max \left\{x_{0}(t): t \in J\right\}=0$. Hence

$$
\begin{aligned}
& \lambda_{0}\left[\max \left\{x_{0}(t): t \in J\right\}+\min \left\{x_{0}(t): t \in J\right\}\right]+\left(1-\lambda_{0}\right) x_{0}(1 / 2) \\
= & \lambda_{0} \min \left\{x_{0}(t): t \in J\right\}+\left(1-\lambda_{0}\right) x_{0}(1 / 2)<0,
\end{aligned}
$$

contrary to (3.5). If $\mu_{0}=0$ and then (3.4) gives $x_{0}=0$. Consequently, $\left(x_{0}, \mu_{0}\right)=(0,0)$, contrary to $\left(x_{0}, \mu_{0}\right) \in \partial \Omega$. The Borsuk antipodal theorem and the Leray-Schauder degree theory lead to $D(\mathcal{I}-\mathcal{H}(0, \cdot, \cdot), \Omega, 0) \neq 0$ and

$$
D\left(\mathcal{I}-\mathcal{F}_{1}, \Omega, 0\right)=D(\mathcal{I}-\mathcal{H}(1, \cdot, \cdot), \Omega, 0)=D(\mathcal{I}-\mathcal{H}(0, \cdot, \cdot), \Omega, 0)
$$

which implies (3.3). 
Finally, let $\mathcal{F}: \bar{\Omega} \rightarrow C^{n}(J) \times R$ be defined by the formula

$$
\begin{aligned}
\mathcal{F}(x, \mu)= & \left((-1)^{n-p-1} \int_{0}^{1} G(t, s) \Phi_{m^{\prime}}\left[\mu \int_{s}^{1} f_{m}\left(r, x(r), \ldots, x^{(n-1)}(r)\right) d r\right] d s,\right. \\
& \max \{x(t): t \in J\}+\min \{x(t): t \in J\}-A+\mu) .
\end{aligned}
$$

We claim that to prove our theorem it is sufficient to verify:

$$
D(\mathcal{I}-\mathcal{F}, \Omega, 0) \neq 0 .
$$

In fact, if $(3.6)$ is true, then there exists a fixed point $(\widehat{x}, \widehat{\mu}) \in \Omega$ of the operator $\mathcal{F}$. Hence

$$
\widehat{x}(t)=(-1)^{n-p-1} \int_{0}^{1} G(t, s) \Phi_{m^{\prime}}\left[\widehat{\mu} \int_{s}^{1} f_{m}\left(r, \widehat{x}(r), \ldots, x^{(n-1)}(r)\right) d r\right] d s
$$

for $t \in J$ and

$$
\max \{\widehat{x}(t): t \in J\}+\min \{\widehat{x}(t): t \in J\}=A .
$$

Moreover, $\widehat{\mu}>0$ since in the case of $\widehat{\mu} \leq 0$ (3.7) and Lemma 2.1 gives for $\widehat{x}(t) \leq 0$ for $t \in J$, so $\max \{\widehat{x}(t): t \in J\}=0$, contrary to (3.8). Therefore (see (3.7)) $\widehat{x}$ is a solution of BVP (2.4), (1.6) with $\mu=\widehat{\mu}$ in (2.4), and for $t \in(0,1)$. So $\min \{\widehat{x}(t): t \in J\}=0$. Then, by (3.8), $\max \{\widehat{x}(t): t \in J\}=A$, and we see that $\widehat{x}$ is a solution of BVP (2.4), (1.6), (1.7).

In order to prove $(3.6)$ we consider the operator $\mathcal{H}:[0,1] \times \bar{\Omega} \rightarrow C^{n}(J) \times R$,

$$
\begin{aligned}
\mathcal{H}(\lambda, x, \mu)= & \left((-1)^{n-p-1} \int_{0}^{1} G(t, s) \Phi_{m^{\prime}}\left[\mu \int_{s}^{1} f_{m}\left(r, x(r), \ldots, x^{(n-1)}(r)\right) d r\right] d s,\right. \\
& \max \{x(t): t \in J\}+\min \{x(t): t \in J\}-\lambda A+\mu)
\end{aligned}
$$

Then, $\mathcal{H}(1, \cdot, \cdot)=\mathcal{F}, \mathcal{H}(0, \cdot, \cdot)=\mathcal{F}_{1}$ and, by $(3.3)$,

$$
D(\mathcal{I}-\mathcal{H}(0, \cdot, \cdot), \Omega, 0) \neq 0
$$

Assume that $\mathcal{H}\left(\lambda_{1}, x_{1}, \mu_{1}\right)=\left(x_{1}, \mu_{1}\right)$ for some $\lambda_{1} \in[0,1]$ and $\left(x_{1}, \mu_{1}\right) \in \partial \Omega$. If $\mu_{1}=0$ then from the equality

$$
x_{1}(t)=(-1)^{n-p-1} \int_{0}^{1} G(t, s) \Phi_{m^{\prime}}\left[\mu_{1} \int_{s}^{1} f_{m}\left(r, x_{1}(r), \ldots, x_{1}^{(n-1)}(r)\right) d r\right] d s,
$$

for $t \in J$, we get $x_{1}=0$, contrary to $\left(x_{1}, \mu_{1}\right)=(0,0) \in \partial \Omega$. let $\mu_{1}<0$. Then (see (3.10)) $x_{1}(t)<0$ on $(0,1)$ and $\max \left\{x_{1}(t): t \in J\right\}=0$, contrary to $\max \left\{x_{1}(t): t \in\right.$ $J\}+\min \left\{x_{1}(t): t \in J\right\}=\lambda_{1} A$. hence $\mu_{1}>0$ and then $x_{1}$ is a solution of BVP (2.5), (1.6) with $\max \{x(t): t \in J\}=\lambda A$. Moreover, by Lemma $2.5,\left\|x_{1}^{(j)}\right\| \leq P$ for $0 \leq j \leq n-1$ and $0<\mu \leq \mu_{*}$. Consequently, $\left(x_{1}, \mu_{1}\right) \notin \partial \Omega$, a contradiction. we have proved $\mathcal{F}(\lambda, x, \mu) \neq(x, \mu)$ for each $\lambda \in[0,1]$ and $(x, \mu) \in \partial \Omega$, and since $\mathcal{H}$ is a compact homotopy,

$$
D(\mathcal{I}-\mathcal{F}, \Omega, 0)=D(\mathcal{I}-\mathcal{H}(1, \cdot, \cdot), \Omega, 0)=D(\mathcal{I}-\mathcal{H}(0, \cdot, \cdot), \Omega, 0)
$$


and then (3.9) gives (3.6), which finishes our proof.

Theorem 3.2. Suppose the assumptions $\left(H_{1}\right)$ be satisfied and $A \in R_{+}$. Then there exists a solution of $B V P(1.5),(1.6),(1.7)$ for each $A \in R_{+}$.

Proof. For each $m \in N$, there exists a solution $x_{m}$ of BVP (2.5), (1.6), (1.7) with a $\mu=\mu_{m}$ by Theorem 3.1. Consider the sequence $\left\{x_{m}\right\},\left\{\mu_{m}\right\}$. By Lemma 2.2, Lemma $2.5,\left\{x_{m}^{(i)}\right\},\left\{\mu_{m}\right\}$ are bounded for $i=0, \ldots, n-1$.

For $t_{1}, t_{2} \in J, t_{2}<t_{1}$,

$$
\begin{aligned}
& \left|x_{m}^{(n-1)}\left(t_{1}\right)-x_{m}^{(n-1)}\left(t_{2}\right)\right| \\
= & \Phi_{m^{\prime}}\left(\left|\int_{0}^{t_{1}} \mu_{m} f_{m}\left(t, x_{m}(t), \ldots, x_{m}^{(n-1)}(t)\right) d t\right|\right) \\
& -\Phi_{m^{\prime}}\left(\left|\int_{0}^{t_{2}} \mu_{m} f_{m}\left(t, x_{m}(t), \ldots, x_{m}^{(n-1)}(t)\right) d t\right|\right) .
\end{aligned}
$$

We can use Lemma 2.6 and obtain that the sequence $\mu_{m} f_{m}\left(t, x_{m}(t), \ldots, x_{m}^{(n-1)}(t)\right)$ is uniformly absolutely continuous on $J$. Moreover by the continuity of $\Phi_{m^{\prime}}$ we have $\left\{x_{m}^{(n-1)}\right\}_{n_{0}}^{\infty}$ is equi-continuous on $J$. The Arzalà-Ascoli theorem guarantees the existence of a subsequence, such that $\left\{x_{m_{k}}\right\},\left\{\mu_{m_{k}}\right\}$ is convergent in $C^{n}(J)$ and $R$ respectively. Let $\lim _{k \rightarrow \infty} x_{m_{k}}=x, \lim _{k \rightarrow \infty} \mu_{m_{k}}=\widehat{\mu}$, then $x \in C^{n-1}(J), x$ satisfies boundary condition (1.6), (1.7) and $0 \leq \widehat{\mu} \leq \mu_{*}$. that

We now prove $(-1)^{n-p-1} x^{(n-1)}(t)>0, t \in[0,1)$. If not, there exists $t_{1} \in(0,1)$ such

$$
(-1)^{n-p-1} x^{(n-1)}(t)>0, t \in\left[t_{1}, 1\right), \quad(-1)^{n-p-1} x^{(n-1)}(t)=0, t \in\left(0, t_{1}\right]
$$

From $(2.3)$ we obtain $x^{(i)}$ has at most one zero $\xi_{j}$ on $\left[0, t_{1}\right]$ for $i=0, \ldots, p-1$. Now from the construction of $f_{m_{k}} \in \operatorname{Car}\left(J \times R^{n-1}\right)$ it follows that there exists a set $\mathcal{M} \in$ $J, \mu(\mathcal{M})=0$ such that $f_{m_{k}}(t, \cdot, \ldots, \cdot)$ are continuous on $R^{n-1}$ for each $t \in J \backslash \mathcal{M}$ which implies that

$$
\lim _{k \rightarrow \infty} \mu_{m_{k}} f_{m_{k}}\left(t, x_{m_{k}}(t), \ldots, x_{m_{k}}^{(n-1)}(t)\right)=\widehat{\mu} f\left(t, x(t), \ldots, x^{(n-1)}(t)\right)
$$

for $t \in\left[0, t_{1}\right] \backslash \mathcal{M}$. By Lemma $2.6\left\{\mu_{m_{k}} f_{m_{k}}\left(t, x_{m_{k}}(t), \ldots, x_{m_{k}}^{(n-1)}(t)\right)\right\}$ is uniformly absolutely continuous on $\left[0, t_{1}\right]$. Then $\widehat{\mu} f\left(t, x(t), \ldots, x^{(n-1)}(t)\right) \in L^{1}\left[0, t_{1}\right]$ and

$$
\lim _{k \rightarrow \infty} \mu_{m_{k}} \int_{t}^{t_{1}} f_{m_{k}}\left(s, x_{m_{k}}(s), \ldots, x_{m_{k}}^{(n-1)}(s)\right) d s=\widehat{\mu} \int_{t}^{t_{1}} f\left(s, x(s), \ldots, x^{(n-1)}(s)\right) d s
$$

for $t \in\left[0, t_{1}\right]$ by Vitali's convergence theorem. Noticing $x_{m_{k}}^{(n-1)}\left(t_{1}\right)$ is bounded, we assume it is convergent, and let $\lim _{k \rightarrow \infty} x_{m_{k}}^{(n-1)}\left(t_{1}\right)=d$. Taking the limit as $k \rightarrow \infty$ in the equality

$$
x_{m_{k}}^{(n-1)}(t)=x_{m_{k}}^{(n-1)}\left(t_{1}\right)-(-1)^{n-k} \Phi_{m^{\prime}}\left(\mu_{m_{k}} \int_{t}^{t_{1}} f_{m_{k}}\left(\tau, x_{m_{k}}(\tau), \ldots, x_{m_{k}}^{(n-1)}(\tau)\right) d \tau\right)
$$


we get

$$
x^{(n-1)}(t)=d-(-1)^{n-k} \Phi_{m^{\prime}}\left(\widehat{\mu} \int_{t}^{t_{1}} f\left(\tau, x(\tau), \ldots, x^{(n-1)}(\tau)\right) d \tau\right)
$$

There are two cases to consider:

Case(i) If $\widehat{\mu}=0 . x^{(n-1)}(t)=0$ for $t \in\left[0, t_{1}\right]$, and the equality $x^{(n-1)}\left(t_{1}\right)=0$ yields $d=0$. Hence $x^{(n-1)}(t)=0$ for $t \in J$, contrary to (3.10).

Case(ii) If $\widehat{\mu}>0$. By (2.4), we have

$$
\left|x_{m_{k}}^{(n-1)}(t)\right| \geq \Phi_{m^{\prime}}\left(\mu_{m_{k}} \int_{t}^{1} \phi(\theta) d \theta\right), k \in N
$$

Letting $k \rightarrow \infty$ in $(3.11)$ we have

$$
\left|x^{(n-1)}(t)\right| \geq \Phi_{m^{\prime}}\left(\widehat{\mu} \int_{t}^{1} \phi(\theta) d \theta\right), t \in J
$$

Hence $\left|x^{(n-1)}(t)\right|>0$ for $t \in[0,1)$, contrary to (3.11). Thus $\left|x^{(n-1)}(t)\right|>0$ for $t \in[0,1)$. So $x^{(i)}(t)>0,0 \leq i \leq p-1$ on $(0,1],(-1)^{2 n-p-i} x^{(i)}(t)>0, p \leq i \leq$ $n-1$ on $[0,1)$. Noticing $\left\{x_{m_{k}}^{(n-1)}(0)\right\}$ is convergent. Let $\lim _{k \rightarrow \infty} x_{m_{k}}^{(n-1)}(0)=\widehat{d}$. Since $\left\{\mu_{m_{k}} f_{m_{k}}\left(t, x_{m_{k}}(t), \ldots, x_{m_{k}}^{(n-1)}\right)\right\}$ is uniformly absolutely continuous on $J$ and

$$
\lim _{k \rightarrow \infty} \mu_{m_{k}} f_{m_{k}}\left(t, x_{m_{k}}(t), \ldots, x_{m_{k}}^{(n-1)}(t)\right)=\mu f\left(t, x(t), \ldots, x^{(n-1)}(t)\right) .
$$

By the Vitali's Convergence theorem to get $\widehat{\mu} f\left(t, x(t), \ldots, x^{(n-1)}(t)\right) \in L_{1}(J)$ and letting $k \rightarrow \infty$ in the equality

$$
x_{m_{k}}^{(n-1)}(t)=x_{m_{k}}^{(n-1)}(0)+(-1)^{n-k} \Phi_{m^{\prime}}\left(\mu_{m_{k}} \int_{0}^{t} f_{m_{k}}\left(s, x_{m_{k}}(s), \ldots, x_{m_{k}}^{n-1}(s)\right) d s\right), t \in J
$$

we get

$$
x^{(n-1)}(t)=\widehat{d}+(-1)^{n-k} \Phi_{m^{\prime}}\left(\widehat{\mu} \int_{0}^{t} f\left(s, x(s), \ldots, x^{n-1}(s)\right) d s\right), t \in J .
$$

If $\widehat{\mu}=0 . x^{(n-1)}(t)=\widehat{d}$ for $t \in J$ and condition $x^{(n-1)}(1)=0$ gives $\widehat{d}=0$. So $x^{(n-1)}(t)=0$ for $t \in J$. Without loss of generality, we suppose $\left\|x^{(i)}\right\|=x\left(\zeta_{i}\right)$.

$$
\left\|x^{(i)}\right\|>\left|\frac{x^{(i-1)}\left(\zeta_{i-1}\right)-x^{(i-1)}(0)}{\zeta_{i-1}}\right|>\left\|x^{(i-1)}\right\|, \quad 0 \leq i \leq p-1
$$

and

$$
\left\|x^{(i)}\right\|>\left|\frac{x^{(i-1)}(1)-x^{(i-1)}\left(\zeta_{i-1}\right)}{1-\zeta_{i-1}}\right|>\left\|x^{(i-1)}\right\|, \quad p \leq i \leq n-1
$$

Thus $\left\|x^{(i)}\right\|>\left\|x^{(i-1)}\right\|>\cdots>\|x\|=A, 0 \leq i \leq n-1$. The fact $x^{(n-1)}(t)=0$ for $t \in J$ contradicts $\left\|x^{(n-1)}\right\|>A$. 
If $\widehat{\mu}>0$ and from (3.13) we see that $x \in A C^{n-1}(J)$ and $x$ satisfies (1.5) a.e. on $J$. We have proved that $x$ is a solution of BVP (1.5)-(1.7) with $\mu=\widehat{\mu}$ in (1.5).

\section{Example}

Example 4.1. Let us consider the following fourth-order boundary value problem

$$
\left\{\begin{array}{l}
\left(\Phi_{m}\left(y^{(3)}(t)\right)\right)^{\prime}=\mu\left[1-t+\sum_{i=0}^{3} q_{i}(t) y_{i}^{-\frac{1}{5}}+g_{0}(t) \sin \left(\Phi_{3}\left(y_{0}\right)\right)^{\frac{1}{2}}+\sum_{i=1}^{3} g_{i}(t) \Phi_{3}\left(y_{i}\right)\right], \\
y(0)=0, \quad y^{\prime}(1)=y^{\prime \prime}(1)=y^{(3)}(1)=0,
\end{array}\right.
$$

with $\max \{y(t): t \in[0,1]\}=A, q_{i} \in L_{\infty}([0,1]), g_{i} \in C[0,1], m=3, p=1$ for $i=0,1,2,3$.

Corresponding to BVP (1.5)-(1.7) we have

$$
f\left(t, y_{0}, y_{1}, y_{2}, y_{3}\right)=1-t+\sum_{i=0}^{3} q_{i}(t) y_{i}^{-\frac{1}{5}}+g_{0}(t) \sin \left(\Phi_{3}\left(y_{0}\right)\right)^{\frac{1}{2}}+\sum_{i=1}^{3} g_{i}(t) \Phi_{3}\left(y_{i}\right)
$$

where $\phi(t)=1-t, \omega_{i}\left(\left|y_{i}\right|\right)=\left|y_{i}\right|^{-\frac{1}{5}}, i=0,1,2,3, g_{0}\left(t, y_{0}\right)=g_{0}(t) \sin \left(\Phi_{3}\left(y_{0}\right)\right)^{\frac{1}{2}}, g_{i}\left(t, y_{i}\right)=$ $g_{i}(t) \Phi_{3}\left(y_{i}\right), i=1,2,3$.

Then for any $A>0$, there exists $\mu_{A}<\mu_{*}=\Phi_{3}\left(\frac{A}{\Gamma}\right)$ such that BVP (4.1) has a solution $y \in A C^{3}([0,1])$.

To see (4.1) has a solution $y \in A C^{3}([0,1])$, we apply theorem 3.2 , It is easy to verify $\left(H_{1}\right)$

$$
\begin{gathered}
\lim _{\left|y_{0}\right| \rightarrow \infty} \sup _{t \in[0,1]} \frac{g_{0}\left(t, y_{0}\right)}{\left(\Phi_{3}\left(\left|y_{0}\right|\right)\right)^{1 / 2}}=0, \\
\lim _{\left|y_{i}\right| \rightarrow \infty} \sup _{t \in[0,1]} \frac{g_{i}\left(t, y_{i}\right)}{\Phi_{3}\left(\left|y_{i}\right|\right)}=\sup _{t \in[0,1]} g_{i}(t) \geq 0, \\
\int_{0}^{1} \omega_{0}(s) d s<\infty \\
\int_{0}^{1} \omega_{i}\left(P_{i}(s)\right) d s<\infty, \quad i=1,2,3
\end{gathered}
$$

hold. So applying Theorem 3.2, for any $A>0$, there exists $\mu_{A}$ such that BVP (4.1) has a solution $y \in A C^{3}([0,1])$.

\section{References}

[1] R. P. Agarwal, Boundary Value Problems for Higher Order Differential Equations, World Scientific, Singapore, 1986.

[2] R. P. Agarwal and D. O'Regan, Right Focal Singular boundary value problems, ZAMM 79 (1999), 363-373. 
[3] R. P. Agarwal, D. O'Regan, V. Lakshmikanam, Singular $(p, n-p)$ focal and $(n, p)$ higher order boundary value problems, Nonlinear Anal. 42(2000), 215-228.

[4] R. P. Agarwal and D. O'Regan, Multiplicity results for singular conjugate focal, and $(N, P)$ problems, J. Differ. Equ. 170(2001), 142-156.

[5] R. P. Agarwal, D. O'Regan, I. Rachunkov and S. Staněk, Two-point higher-order BVPs with singularities in phase variables, Comput. Math. Appl. 46(2003), 1799-1826.

[6] R. P. Agarwal, D. O'Regan and S. Staněk, Singular Lidstone boundary value problem with given maximal values for solutions, Nonlinear Anal. 55(2003), 859-881.

[7] D. Anderson, R. Avery and A. Peterson, Positive solutions to a discrete focal boundary value problem, J. Comput. Appl. Math. 88(1998), 103-118.

[8] D. Anderson and R. I. Avery, Multiple positive solutions to a third-order discrete focal boundary value problem, Comput. Math. Appl. 42(2001), 333-340.

[9] D. R. Anderson nad J. R. Davis, Multiple solutions and eigenvalues for third-order right focal boundary value problems, J. Math. Anal. Appl. 267(2002), 135-157.

[10] F. Atici and A. Peterson, Bounds for positive solutions for a focal boundary value problem, Comput. Math. Appl. 36(1998), 99-107.

[11] R. G. Bartle, A Modern Theory of Integration, AMS, Providence, RI, 2001.

[12] K. Deimling, Nonlinear Functional Analysis, Spinger, Berlin, Heidelberg, 1985.

[13] Johnny Henderson and William Yin, Singular $(k, n-k)$ boundary value problems between conjugate and right focal, J. Comput. Appl. Math. 88(1998), 57-69.

[14] I. P. Natanson, Theorie der Funktionen einer reelen Veränderlichen, Akademie-Verlag, berlin, 1969.

[15] P. J. Y. Wong and R. P. Agarwal, Multiple positive solutions of two-point right focal boundary value problems, Math. Comput. Modelling 28(1998), 41-49.

School of Science, Beijing University of Posts and Telecommunications, Beijing 100876, P.R. China.

Department of Applied Mathematics, Beijing Institute of Technology, Beijing 100081, P.R. China.

E-mail: tianyu2992@163.com

Department of Applied Mathematics, Beijing Institute of Technology, Beijing 100081, P.R. China.

E-mail: gew@bit.edu.cn 